\title{
Análisis del bienestar en la vejez según la edad
}

\author{
Juan Carlos Meléndez Morala , José Manuel Tomás Miguel ${ }^{b}$ y Esperanza Navarro Pardo ${ }^{a}$ \\ aDepartamento de Psicología Evolutiva y de la Educación. Universitat de Valencia. Valencia. España. \\ bepartamento de Metodología de las Ciencias del Comportamiento. Universitat de Valencia. Valencia. España.
}

\begin{abstract}
Introducción: el objetivo de este trabajo es conocer cómo el bienestar evoluciona con la edad una vez llegados al período de la vejez y si existen cambios en esta variable tan relacionada con la calidad de vida.

Material y método: se aplicó a una muestra de 169 personas de más de 65 años la Life Satisfaction Index (LSI) y la escala de bienestar de Ryff; se realizaron regresiones lineales simples y curvilíneas para ver su relación con la edad.

Resultados: las correlaciones de Pearson mostraron que parece existir una relación de disminución, tanto del bienestar como indicador global, y de tres de sus dimensiones, con la edad. Por otra parte, la satisfacción vital muestra como mejor ajuste el cuadrático, lo que indica que, mientras en los primeros años de la vejez efectivamente hay una disminución, a partir de los 85 años se produce un repunte de la satisfacción vital.

Conclusiones: el aumento de estrategias adaptativas de carácter acomodativo se presupone como causa del descenso de los indicadores más propios del bienestar psicológico. Por otra parte, el aumento de la longevidad y su relación con variables de salud, competencia y relaciones pueden estar generando un aumento de la satisfacción por las características diferenciales de la propia muestra.
\end{abstract}

\section{Palabras clave}

Bienestar. Satisfacción vital. Vejez. Envejecimiento con éxito.

\section{An analysis of well-being and age in the elderly}

Introduction: well-being is a key construct for understanding quality of life. The aim of the present study was to analyze how well-being changes in old age and to determine if there are any modifications in this variable during this period.

Material and methods: the LIS and EBR scales were applied to a sample of 169 persons aged more than 64 years. Linear and curvilinear regressions were estimated to understand the associations among age, well-being and life satisfaction.

Correspondencia: Dr. J.C. Meléndez Moral.

Departamento de Psicología Evolutiva y de la Educación. Facultad de Psicología. Universitat de Valencia.

Av. Blasco Ibáñez, 21. 46010 Valencia. España.

Correo electrónico: melendez@uv.es

Recibido el 26-6-2007; aceptado el 5-12-2007.
Results: pearson correlations showed statistically significant negative relations among age and environmental mastery, personal growth, purpose in life, and overall well-being. However, life satisfaction showed a curvilinear, quadratic relation with age, revealing that this dimension decreases in the first few years of old age but increases again after the age of 85 years.

Conclusions: the decrease in several well-being factors could be caused by the increase in adaptive accommodation strategies. Increased longevity, together with better health, competence and relationships could potentially explain the increase in life satisfaction among the oldest population.

\section{Key words}

Well-being. Life satisfaction. Elderly. Successful ageing.

\section{INTRODUCCIÓN}

Desde la psicología del desarrollo la edad es planteada como un marcador de cambio y transformación de la conducta humana, tanto en sus aspectos externos y visibles como en los internos y no directamente perceptibles. Así, la edad se convierte en una variable básica a lo largo de la cual pueden ordenarse e investigarse los cambios evolutivos relacionados con el período del desarrollo humano en el que la persona se encuadra.

Partimos de la calidad de vida ${ }^{1}$ en la vejez como un concepto multidimensional que comprende componentes tanto objetivos como subjetivos, de tal manera que su evaluación incluye 3 dimensiones básicas: funcionamiento psicológico, funcionamiento social y funcionamiento físi$\mathrm{CO}^{2,-4}$; pero, además, también se deben tener en cuenta las impresiones subjetivas como la satisfacción vital y los factores ambientales, es decir, la percepción que tiene la persona de estas dimensiones ${ }^{5,6}$.

Aunque en principio resultaría lógico pensar que la satisfacción vital podría ser uno de los indicadores del funcionamiento psicológico, lo cierto es que la satisfacción con la vida se considera a otro nivel, no como un elemento o indicador de una de las dimensiones, sino como un indicador macro, en el que participan, en mayor o menor medida, las 3 dimensiones señaladas ${ }^{7}$.

De este modo, la aproximación orientada al análisis del bienestar en diferentes edades y sus posibles cambios parecen fundamentales, sobre todo si el bienestar es un marca- 
dor de la calidad de vida y ésta es una de las bases del envejecimiento con éxito.

En lo referente al análisis del bienestar, se han mantenido dos líneas de trabajo paralelas centradas en lo que se ha definido como bienestar subjetivo y como bienestar psicológico.

El bienestar subjetivo es cercano a conceptos de larga tradición en la psicología como el de satisfacción vital, y hace referencia a la experiencia subjetiva de felicidad. Este tipo de bienestar incluye un componente emocional o afectivo, relacionado con los sentimientos de placer y displacer que experimenta la persona y que comprendería el concepto de felicidad, y un componente de carácter cognitivo referido al juicio que merece a la persona su trayectoria evolutiva, es decir, lo que tradicionalmente se ha denominado satisfacción vital ${ }^{8,9}$. Este tipo de bienestar con respecto a su relación con la edad y cómo evoluciona parece mostrar una aparente estabilidad ${ }^{10,11}$.

La segunda línea de trabajo es la que se ha centrado en el denominado bienestar psicológico; el concepto se sitúa en el proceso y consecución de los valores que nos hacen sentir vivos y auténticos, que nos hacen crecer como personas, y no tanto en las actividades que nos dan placer o nos alejan del dolor ${ }^{12}$.

Desde una perspectiva quizá más amplia ${ }^{13-15}$, parece ser que una caracterización más ajustada es definirlo como un esfuerzo por perfeccionarse y conseguir la realización del propio potencial. Así, el bienestar psicológico estaría relacionado con tener un propósito en la vida, con que la vida adquiera significado para uno mismo, con los desafíos y con un cierto esfuerzo por superarlos y conseguir metas valiosas, mientras que el bienestar subjetivo tendría que ver más con sentimientos de relajación, ausencia de problemas y presencia de sensaciones positivas.

Respecto a la trayectoria evolutiva de las medidas del bienestar psicológico ${ }^{14}$, algunas dimensiones muestran claros efectos de la edad, mientras otras permanecen estables $^{16}$. Así, de las dimensiones señaladas por Ryff y Keyyes ${ }^{13}$ que permanecen relativamente estables, encontramos la autoaceptación y las relaciones positivas con los otros. Respecto al resto de dimensiones, el dominio del ambiente tiende a ser más alto en las personas mayores y de mediana edad que en los jóvenes, pero permanece estable de la mediana edad a la vejez; un patrón similar sigue la dimensión autonomía, aunque en este caso el crecimiento de la juventud a la mediana edad es menos acusado, y, por último, las restantes 2 dimensiones, propósito en la vida y crecimiento personal, típicas del bienestar psicológico, muestran una disminución en los diferentes momentos vitales estudiados, que es mayor al comparar personas de mediana edad y personas mayores.

Del mismo modo ${ }^{17}$, investigaciones posteriores muestran la misma tendencia con respecto a la evolución de la edad. Si bien se puede observar un cierto descenso en las puntuaciones de todas las dimensiones según va aumentando la edad, sólo aquellas que son propias del bienestar psicológico muestran diferencias significativas.
Debemos tener en cuenta que el envejecimiento implica una reducción de los recursos personales de acción y de las opciones evolutivas disponibles. Así, existe una tendencia evolutiva, en la vejez, hacia un tipo de estrategias adaptativas sustentadas en mayor medida en la acomodación a las nuevas situaciones ${ }^{18}$ y no tanto en la asimilación. De este modo, este tipo de estrategias se convierte en un sistema optimizador y compensador de los recursos del desarrollo.

De este modo, los mayores tienden bien a reajustar sus metas manteniendo tanto lo ya conseguido como lo disponible y posible, bien a sustituir sus objetivos por otros más realistas, lo cual ayuda a optimizar los recursos y a tener un mayor control de la situación. En consecuencia, y mediante este tipo de estrategias, logran generar un ajuste de las preferencias y de las metas que, aun no siendo intencional, hace que el sujeto se adapte adecuadamente a la nueva situación, con lo que palía la percepción de las restricciones situacionales o unos recursos personales insuficientes para conseguir metas antes valoradas.

En este sentido, también hemos de tener en cuenta que la reducción del tiempo que queda para conseguir las metas es un elemento fundamental en el reajuste de éstas, ya que modifica las expectativas futuras y lleva a establecer metas a más corto plazo y modestas. Los mayores intentan primordialmente conservar las cosas buenas, todavía disponibles y posibles en su presente y, en este sentido, envejecer, a través de estos movimientos de acomodación, deja de convertirse en una cuestión de ganar y alcanzar nuevos estados para pasar a ser fundamentalmente una cuestión de no perder lo que ya se tiene, manteniéndose ante un incierto futuro. También hemos de pensar que en muchos casos los mayores ya están disfrutando de las metas conseguidas a través de los años, con lo que mantenerlas se convierte en algo con sentido?.

Como objetivo de este trabajo nos interesa conocer cómo el bienestar y la satisfacción vital evolucionan con la edad una vez llegados al período de la vejez y si existen cambios en estas variables tan relacionadas con la calidad de vida.

En consecuencia, y siguiendo los resultados de las investigaciones anteriormente citadas, planteamos como primera hipótesis que existirá una relación inversa entre la edad y las dimensiones típicas del bienestar psicológico, a más edad menos bienestar psicológico, mientras que las demás dimensiones mantendrán una cierta estabilidad. Como segunda hipótesis, planteamos que la satisfacción vital se mantendrá estable con la edad.

\section{MATERIAL Y MÉTODOS}

\section{Diseño}

El diseño de investigación empleado es transversal y de encuesta. Se recogieron las respuestas de una muestra de 
personas mayores de 65 años en un momento temporal de una serie de características vitales y psicológicas.

\section{Muestra}

La muestra estaba compuesta por 169 personas de más de 65 años, jubiladas, de la Comunidad Valenciana (España); los criterios de inclusión fueron: tener 65 años o más, estar jubiladas y no estar institucionalizadas. El muestreo fue de tipo incidental. La recogida de datos se realizó de manera directa e individual entre enero y mayo de 2007; previamente se obtuvo el consentimiento informado de los participantes en el estudio.

Las edades estaban comprendidas entre los 65 y los 94 años; la media fue de 75,4 años y la desviación típica, de 7,11 . Respecto al sexo, un $40,8 \%$ eran varones y un $59,2 \%$, mujeres. En lo referente al estado civil, el 50,3\% estaban casados, un $40,8 \%$ eran viudos y un $8,9 \%$ se encontraba en otras situaciones (solteros, divorciados, etc.). Respecto al nivel educativo, el 31,5\% no tenía estudios; el 53\% tenía estudios básicos, el 10,1\%, estudios medios, y el 5,4\%, estudios superiores. Un $88,2 \%$ vivía en casa propia y un $11,8 \%$, en casa de familiares.

\section{Medidas}

Para la recogida de datos de esta investigación, se utilizó una plantilla con diferentes elementos sociodemográficos, así como la Life Satisfaction Index (LSI-A) ${ }^{19}$, en la versión en castellano desarrollada por Stock et al ${ }^{20}$, de 20 ítems, con respuesta tipo Likert, y la escala de bienestar psicológico de Ryff $(E B R)^{14,17,21}$, de la que entre sus diferentes versiones se seleccionó la de 54 ítems, siguiendo la recomendación de la propia autora en una comunicación personal y que en el contexto español se ha utilizado con resultados moderadamente satisfactorios ${ }^{9,16,22}$. La escala en su versión original está compuesta por 6 factores, que se presentan a continuación, así como la definición de una puntuación alta en cada uno de ellos:

- Autonomía: se es independiente y autodeterminado, capaz de resistir presiones sociales para pensar y actuar de ciertos modos, se regula la conducta internamente y las autoevaluaciones se realizan desde estándares personales.

- Dominio del ambiente: se posee sentido de dominio y competencia manejando el ambiente, se controlan múltiples conjuntos de actividades externas, haciendo un uso efectivo de las oportunidades del contexto y siendo capaz de elegir o crear contextos adaptados a las necesidades y valores personales.

- Autoaceptación: posee una actitud positiva hacia sí mismo, reconociendo y aceptando los múltiples aspectos del yo, incluyendo lo negativo y lo positivo; además existe un sentimiento positivo hacia lo ya vivido.

- Relaciones positivas con otros: mantiene relaciones interpersonales cercanas, satisfactorias, de mutua con- fianza, se preocupa por el bienestar de otros, y es capaz de desarrollar empatía, afecto, intimidad y desde la comprensión del dar y recibir de las relaciones humanas.

- Crecimiento personal: se mantiene el sentimiento de desarrollo continuado y de mejora a través del tiempo, ve el yo en expansión y crecimiento, abierto a nuevas experiencias; sentido de desarrollo del propio potencial y de cambiar en modos que reflejan más autoconocimiento y efectividad.

- Propósito en la vida: tiene metas en la vida y sentido de dirección, sentimiento de significado en el presente y en el pasado; mantiene creencias que proporcionan significado a la vida.

\section{Análisis}

Todos los análisis se han realizado mediante el programa SPSS versión 14. Se han realizado regresiones lineales simples, regresiones curvilíneas, en concreto con ajustes logarítmico, cuadrático y cúbico. Aunque las regresiones curvilíneas planteadas no agotan, obviamente, las posibilidades de relaciones curvilíneas entre variables y, por tanto, el no ajuste de estos 3 modelos no implicaría necesariamente que la relación no es curvilínea. Resulta evidente que se debe tomar una decisión sobre el número de modelos curvilíneos a probar, y éstos son los más utilizados en psicología ${ }^{23}$.

\section{RESULTADOS}

En primer lugar se probaron relaciones de tipo lineal, las más parsimoniosas, entre la edad y las medidas de bienestar y satisfacción vital empleadas. Esto es, si existe algún patrón de aumento o disminución de los factores de bienestar y la satisfacción con la edad. En la tabla 1 se pue-

Tabla 1. Correlaciones de Pearson entre la edad y las diferentes variables estudiadas

\begin{tabular}{|l|c|c|}
\hline & \multicolumn{2}{c|}{ Edad } \\
& Correlación & p \\
\hline Autonomía & $-0,039$ & 0,617 \\
\hline Dominio del ambiente & $-0,256^{\mathrm{a}}$ & 0,001 \\
\hline Autoaceptación & $-0,027$ & 0,723 \\
\hdashline Relaciones positivas con otros & $-0,114$ & 0,139 \\
\hline Crecimiento personal & $-0,293^{\mathrm{a}}$ & 0,000 \\
\hdashline Propósito en la vida & $-0,261^{\mathrm{a}}$ & 0,001 \\
\hline Bienestar (EBR) & $-0,224^{\mathrm{a}}$ & 0,003 \\
\hline Satisfacción vital (LSI) & $0,175^{\mathrm{b}}$ & 0,023 \\
\hline
\end{tabular}

aLa correlación es significante al 0,01 (bilateral).

bLa correlación es significante al 0,05 (bilateral).

EBR: escala de bienestar psicológico de Ryff; LSI: Life Satisfaction Index. 
Tabla 2. Coeficientes de correlación simples y múltiples al cuadrado para los modelos lineal, logarítmico, cuadrático y cúbico estimados para predecir bienestar y sus componentes y la satisfacción vital, en función de la edad

\begin{tabular}{|c|c|c|c|c|}
\hline \multirow[b]{2}{*}{ Criterios } & \multicolumn{4}{|c|}{ Edad } \\
\hline & $\mathrm{R}^{2}$ lineal & $\mathbf{R}^{2}$ logarítmica & $\mathrm{R}^{2}$ cuadrática & $R^{2}$ cúbica \\
\hline Autonomía & 0,001 & 0,002 & 0,003 & 0,004 \\
\hline Dominio del ambiente & $0,066^{*}$ & 0,066 & 0,066 & 0,066 \\
\hline Autoaceptación & 0,001 & 0,001 & 0,024 & 0,025 \\
\hline Relaciones positivas con otros & 0,013 & 0,014 & 0,27 & 0,027 \\
\hline Crecimiento personal & $0,086^{*}$ & 0,089 & 0,097 & 0,097 \\
\hline Propósito en la vida & $0,068^{*}$ & 0,071 & 0,081 & 0,081 \\
\hline Bienestar (EBR) & $0,040^{*}$ & 0,043 & 0,064 & 0,065 \\
\hline Satisfacción vital (LSI) & 0,031 & 0,034 & $0,078^{\star}$ & 0,078 \\
\hline
\end{tabular}

EBR: escala de bienestar psicológico de Ryff; LSI: Life Satisfaction Index.

El asterisco indica qué modelo (lineal, logarítmico, cuadrático y cúbico) representa mejor las relaciones entre predictor y criterio. En aquellos criterios que no hay asterisco, ninguno de los modelos ajusta $(p>0,05)$.

den observar las correlaciones de Pearson que muestran que, efectivamente, en cuatro de las medidas de bienestar, incluido el bienestar global, parece existir una relación de disminución del bienestar con la edad, así como también una disminución en la satisfacción vital.

No obstante, la edad podría presentar relaciones curvilíneas con el bienestar y la satisfacción vital, y esto se puso a prueba mediante la estimación de distintos modelos lineales y no lineales. En concreto, en nuestro caso, se aplicaron los modelos logístico, cuadrático y cúbico para predecir mediante la edad los criterios de bienestar y satisfacción vital. La tabla 2 ofrece los resultados de ajuste de estos modelos lineales y no lineales.

En este sentido, destacamos dos resultados. El primero es que aquellas variables que no presentaban relación lineal estadísticamente significativa en la tabla 1, tampoco presentaron ninguno de los tipos de relaciones curvilíneas exploradas. De las variables de bienestar y satisfacción que sí se encontraban relacionadas linealmente, evidentemente la relación lineal significativa permanece, y solamente en uno de los casos, el de satisfacción vital, encontramos que el modelo cuadrático mejora sustancialmente el ajuste, pues duplica el porcentaje de variancia explicada del modelo lineal.

La figura 1 ilustra, mediante diagramas de dispersión con líneas ajustadas, las relaciones significativas encontradas entre las medidas de bienestar y satisfacción vital con la edad. Puede verse que en todas las medidas de bienestar el modelo lineal es el que mejor representa a los datos, mientras que existe una ganancia relevante mediante el modelo cuadrático solamente para la satisfacción vital.

\section{DISCUSIÓN}

Tal y como indicábamos, el objetivo básico de este trabajo fue determinar si existe un patrón que relacione la edad con las medidas de bienestar y satisfacción vital en adultos de 65 o más años. Así, y tal y como se ha podido observar en el apartado de resultados, observamos que existe una ligera tendencia al descenso tanto la satisfacción vital como del propio bienestar; este descenso es característico en las medidas más propias del bienestar psicológico.

En relación con la primera de las hipótesis planteadas, se ha observado un ligero pero claro descenso en las dimensiones crecimiento personal y propósito en la vida según aumentaba la edad, al igual que se puede observar en otros trabajos ${ }^{14,16}$. Este descenso puede tener una doble explicación; por una parte, mientras que la esperanza de vida es cada vez mayor, el contexto sigue ofreciendo oportunidades reducidas para este grupo de edad, lo cual no favorecería el crecimiento ni el desarrollo personal. Por otra parte, y tal y como se indicaba, el descenso de estas dimensiones podría ser la consecuencia de un proceso de adaptación acomodativa, en la que los ancianos compensan la pérdida de posibilidades reajustando sus metas.

De forma paralela también se ha observado un descenso en la dimensión dominio del ambiente, que tiene que ver con el uso efectivo de oportunidades creando contextos adaptados. En la misma línea de lo ya expuesto, el mayor uso de estrategias acomodativas por parte de las personas mayores implica que la persona modifica sus objetivos, bien reduciendo su nivel de aspiración y desechando ciertos objetivos para establecer otros, bien estableciendo parámetros alternativos de comparación para evaluar su situación actual. En contraste con los mecanismos de asimilación, la acomodación no implica una acción intencional dirigida a una meta, sino que es un proceso más bien automático?.

Por otra parte y respecto a la segunda de las hipótesis, llama la atención el tipo de ajuste cuadrático que mantiene la satisfacción vital con la edad. De este modo, aunque en otros trabajos $9,19,20$ la satisfacción vital mantiene una 

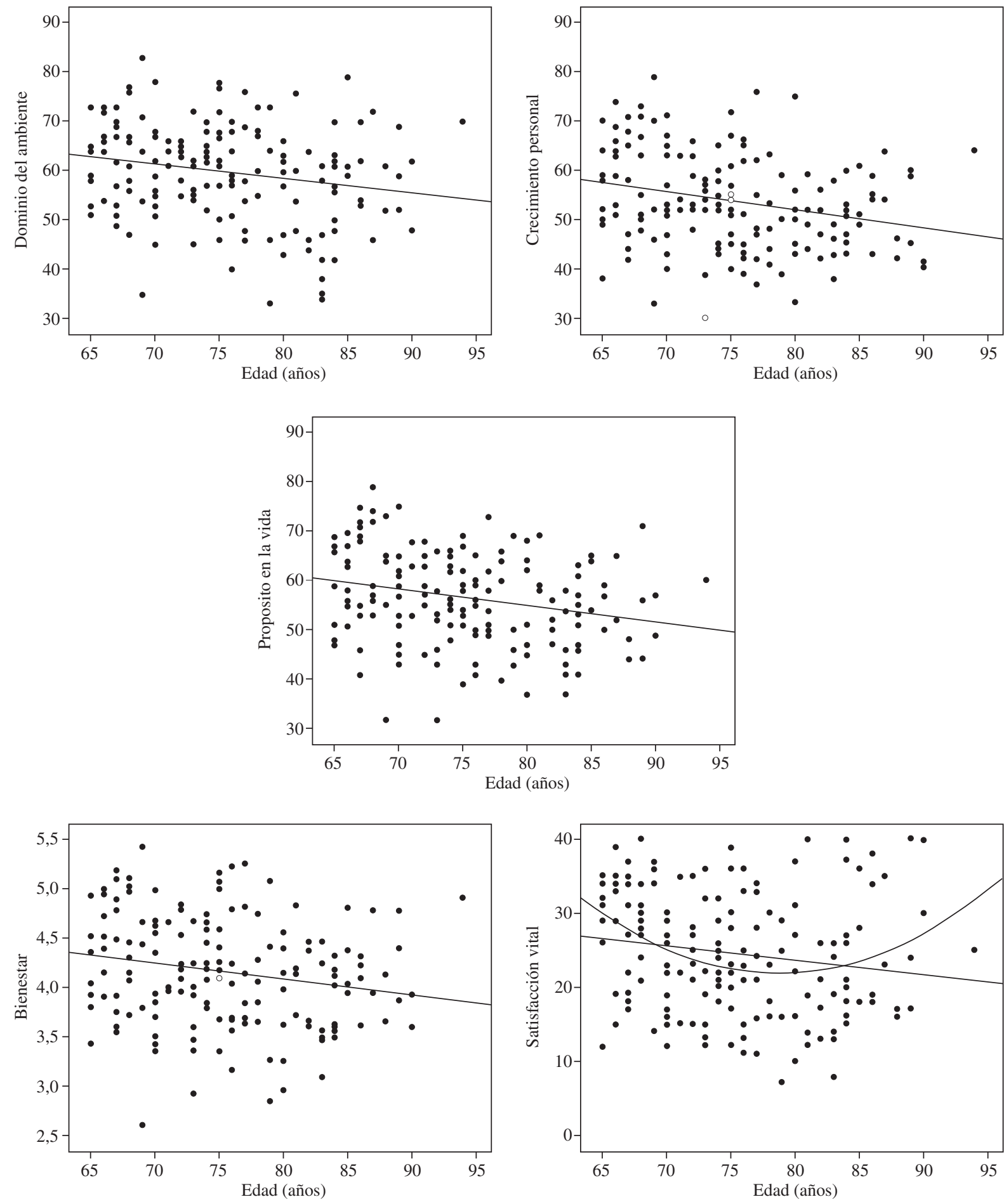

Figura 1. Diagramas de dispersión entre las medidas de bienestar y satisfacción vital con la edad.

tendencia descendente según va aumentando la edad, que era la hipótesis planteada, según nuestros datos se observa un cierto repunte en los últimos años.
A este respecto, debemos tener en cuenta que los datos de las pirámides de población indican que cada vez existen más personas nonagenarias, pero además, cada vez los 
ancianos que llegan a estas edades lo hacen con mejores capacidades. Así, parece existir una relación entre la longevidad y variables tales como el mantenimiento de un buen bienestar, de una adecuada competencia para vivir de forma independiente y del mantenimiento de unas relaciones personales positivas ${ }^{24}$. En esta misma línea, algunos trabajos ${ }^{25,26}$ muestran la importancia del bienestar como una variable predictora de la longevidad. Por otra parte, en una investigación de 2.282 sujetos de más de 65 años ${ }^{27}$, se evaluó el estado de salud y el estado emocional; s concluyó que la experiencia de emociones positivas protegía a las personas mayores de los efectos más negativos del envejecimiento y de la incapacidad y, lo más importante, predecía con éxito quiénes vivirían y quiénes morirían.

Por último, se debe indicar como limitación a nuestro trabajo que los estudios de carácter transversal no nos permiten distinguir entre los efectos del propio desarrollo y los efectos generacionales o de cohorte. De este modo, y de cara a trabajos posteriores, nos parece importante ampliar el rango de edad a grupos anteriores a los 65 años, de manera que se pueda observar si existen diferencias con otros grupos de edad y, sobre todo, determinar si cuando llega el período de la jubilación se produce una modificación sustancial del bienestar frente a los niveles desarrollados en edades anteriores. En este sentido, el trabajo de Ryff $^{14}$ con jóvenes, adultos y mayores y, en menor grado, pero en nuestro contexto, el de Triadó 9,17 , ofrecen un buen ejemplo. Adicionalmente, los diseños de tipo longitudinal serían los más convenientes para resolver estas limitaciones.

\section{BIBLIOGRAFÍA}

1. Yanguas JJ. Análisis de la calidad de vida relacionada con la salud en la vejez desde una perspectiva multidimensional. Madrid: IMSERSO, Colección Estudios: Serie Personas Mayores; 2006.

2. Reig A. Quality of life. En: Fernández Ballesteros R, editor. Encyclopedia of Psychological Assessment. London: Sage; 2003; p. 800-5.

3. Birren JE, Schaie KW. Handbook of the psychology of aging. 5th ed. San Diego: Academic Press; 2001.

4. Izal M, Montorio I. Gerontología conductual. Bases para la intervención y ámbitos de aplicación. Madrid: Síntesis; 1999.

5. Terol MC, López-Roig S, Rodríguez-Marín J, Pastor MA, Mora M, Martín-Aragón M, et al. Diferencias en la calidad de vida: un estudio longitudinal de pacientes de cáncer recibiendo tratamiento de quimioterapia. Anales de Psicología. 2000;16:111-22.
6. Iglesias-Souto PM, Dosil A. Algunos indicadores de percepción subjetiva implicados en la satisfacción del residente mayor. Propuesta de una escala de medida. Rev Esp Geriatr Gerontol. 2005;40:85-91.

7. Cabañero MJ, Richard M, Cabrero J, Orts I, Reig A, Tosal B. Fiabilidad y validez de la escala de satisfacción con la vida de Diener en una muestra de mujeres embarazadas y puérperas. Psicothema. 2004;16:448-55.

8. Diener E, Lucas RE. Personality and sujective well-being. En: Kahneman D, Diener E, Schwarz N, editors. Well-being: The foundations of hedonic psychology. New York: Russell Sage; 1999. p. 213-27.

9. Triadó MC. Envejecer en entornos rurales. Madrid: IMSERSO, Estudios I+D+I, n. ${ }^{\circ} 19 ; 2003$.

10. Mroczek DK, Kolarz CM. The effect of age on positive and negative affect: A developmental perspective on happiness. J Pers Soc Psychol. 1998;75:1333-49.

11. Okun MA, Stock WA. Correlates and components of subjective wellbeing among the elderly. J Appl Gerontol. 1987;6:95-112.

12. Ryan RM, Deci EL. On happiness and human potentials: A review of research on hedonic and eudaimonic wellbeing. Ann Rev Psychol. 2001;52:141-66.

13. Ryff C, Keyes C. The Structure of Psychological Well-Being Revisited. J Pers Soc Psychol. 1995;69:719-27.

14. Ryff CD. Happines is everything: or is it? Explorations on the meaning of psychological well-being. J Pers Soc Psychol. 1989;57:1069-81.

15. Ryff CD. Optimizing well-being: The empirical encounter of two traditions. J Person Soc Psychol. 2002;82:1007-22.

16. Villar F, Triadó C, Resano CS, Osuna MJ. Bienestar, adaptación y envejecimiento: cuando la estabilidad significa cambio. Revista Multidisciplinar de Gerontología. 2003;13:152-62.

17. Villar F, Triadó C, Solé C, Osuna MJ. La medida del bienestar en personas mayores: adaptación de la Escala de Ryff. Revista de Psicología General y Aplicada. 2005;58:347-64.

18. Brandtstädter J, Renner G. Tenacious goal pursuit and flexible goal adjustment: Explication and age-related analysis of assimilative and accommodative strategies of coping. Psychology and Aging. 1990;5:5867.

19. Neugarten BL, Havighurst RJ, Tobin SS. The measurement of life satisfaction. J Gerontol. 1961;16:134-43.

20. Stock WA, Okun MA, Gómez J. Subjective well-being measures: Reliability and validity among Spanish elders. Int J Aging Human Develop. 1994;38:221-35

21. Triadó C, Villar F, Solé C, Celdrán M. Construct validity of Ryff's scale of psychologial well-being in Spanish older adults. Psychological Reports. 2007; 100:1151-64.

22. Navarro E, Meléndez JC, Tomas JM. Influencia de la variable edad en el bienestar de las personas mayores. 49. ${ }^{\circ}$ Congreso de la Sociedad Española de Geriatría y Gerontología; 6-9 de junio de 2007, Palma de Mallorca, España.

23. Pedhazur EJ, Schmelkin LP. Measurement Design and Analysis: An Integrated Approach. Hillsdale: Lawrence Erlbaum Associates; 1991.

24. Domínguez G, Zaldívar D, Pérez J. Caracterización del estilo de vida de individuos con longevidad satisfactoria. Psicología para América Latina. 2006. Disponible en: http://psicolatina.org/Seis/longevidad.html

25. Vecina ML. Emociones positivas. Papeles del Psicólogo. 2006;1:9-17.

26. Lyubomirsky S, King L, Diener E. The Benefits of Frequent Positive Affect: Does Happiness Lead to Success? Psychol Bull. 2005;131:803-55.

27. Ostir G, Markides K, Black S, Goodwin J. Emotional well-being predicts subsequent functional independence and survival. J Am Geriatr Soc. $2000 ; 48: 473-8$. 